\title{
A deep survey of the low-surface-brightness radio sky
}

\section{Ravi Subrahmanyan*}

Raman Research Institute, C.V. Raman Avenue, Sadashivanagar, Bangalore 560080, India ATNF-CSIRO, PO Box 76, Epping NSW 1710, Australia

E-mail: rsubrahmerri.res.in

\section{R. D. Ekers}

ATNF-CSIRO, PO Box 76, Epping NSW 1710, Australia

E-mail: Ron.Ekers@csiro.au

\section{Lakshmi Saripalli}

Raman Research Institute, C.V. Raman Avenue, Sadashivanagar, Bangalore 560080, India ATNF-CSIRO, PO Box 76, Epping NSW 1710, Australia

E-mail: Isaripalerri.res.in

\section{E. M. Sadler}

School of Physics, University of Sydney, NSW 2006, Australia

E-mail: ems@physics.usyd.edu.au

We have made a radio survey - the Australia Telescope Low Brightness Survey (ATLBS)—of 8.4 square degrees sky area, using the Australia Telescope Compact Array in the 20-cm band, in an observing mode designed to provide wide-field images with exceptional sensitivity in surface brightness, and thereby explore a new parameter space in radio source populations. The goals of this survey are to quantify the distribution in angular sizes, particularly at weak surface brightness levels: this has implications for the confusion in deep surveys with the SKA. The survey is expected to lead to a census of the radio emission associated with low-power radio galaxies at redshifts $1-3$, without any missing extended emission, and hence a study of the cosmic evolution of low-power radio galaxies to higher redshift and a comprehensive study of the AGN feedback during the intense black hole growth phase during this redshift range.

From planets to dark energy: the modern radio universe

October 1-5 2007

University of Manchester, Manchester, UK

\footnotetext{
* Speaker.
} 


\section{Introduction}

Radio continuum surveys are characterised by an observing frequency and a limiting flux density at which the survey is deemed to be more or less complete. An important aspect which is often overlooked is the angular resolution (or, more precisely, the spatial frequency coverage in the case of surveys made using interferometers) of the survey telescope and the variation in the flux sensitivity with the angular size of the source. Single-dish radio telescopes have large beams and, therefore, their sensitivity is limited by confusion. Interferometer surveys with good flux sensitivity are 'blind' to extended source components and may miss sources with low surface brightness. As a consequence, population studies of extragalactic radio sources are incomplete in their understanding of the low surface brightness sources.

Table 1 lists existing surveys that have been made with particularly good surface brightness sensitivity. To compare surveys we have assumed a frequency dependence $S_{v} \propto v^{-1}$ of the flux density $S_{v}$ with frequency $v$, typical for extended components of extragalactic radio sources. Additionally, we have assumed that the extended source sizes exceed the beam (an optimistic assumption for the low resolution surveys). The surface brightness detection limit equivalent at the frequency of $1.4 \mathrm{GHz}$, which are listed in the last column of the table, correspond to 5- $\sigma$ limits in all cases except the 6C survey, which is confused, and for which an optimistic limit to the 151-MHz flux density for reliable detection of extended sources is $1 \mathrm{Jy}$ (Saunders et al. 1987).

As may be seen from Table 1, none of these surveys could have detected sources with surface brightnesses below a few mJy $\operatorname{arcmin}^{-2}$. Deep small-area radio surveys like the HDF-S and the Phoenix deep fields recently observed with the ATCA reach surface-brightness levels of 0.3$0.4 \mathrm{mJy} \operatorname{arcmin}^{-2}$, as do similar surveys made with the VLA with arcsec resolution and $\mu \mathrm{Jy}$ flux sensitivity. These surveys can detect extended emission from high-redshift objects, but because the total area surveyed is small, they cover too small a volume to give a fair sample of the low-surfacebrightness radio source population.

\begin{tabular}{lccccc}
\hline Survey & Telescope & Frequency & $\begin{array}{c}1-\sigma \\
\text { RMS noise }\end{array}$ & Beam FWHM & $\begin{array}{c}\text { Surface brightness } \\
\left(\mathrm{mJy} / \mathrm{arcmin}^{2}\right)\end{array}$ \\
\hline \hline 6C & Cambridge & $151 \mathrm{MHz}$ & $20 \mathrm{mJy}$ & $4.2^{\prime} \times 4.2^{\prime} \operatorname{cosec} \delta$ & 6 \\
$7 \mathrm{C}$ & Cambridge & $151 \mathrm{MHz}$ & $15 \mathrm{mJy}$ & $70^{\prime \prime} \times 70^{\prime \prime} \operatorname{cosec} \delta$ & 6 \\
WENSS & WSRT & $326 \mathrm{MHz}$ & $3.6 \mathrm{mJy}$ & $54^{\prime \prime} \times 54^{\prime \prime} \operatorname{cosec} \delta$ & 5 \\
B2 & Bologna & $408 \mathrm{MHz}$ & $50 \mathrm{mJy}$ & $5^{\prime} \times 4^{\prime} \sec (\mathrm{ZA})$ & 3.6 \\
B3 & Bologna & $408 \mathrm{MHz}$ & $20 \mathrm{mJy}$ & $2.6^{\prime} \times 4.8^{\prime} \sec (\mathrm{ZA})$ & 2.3 \\
SUMSS & MOST & $843 \mathrm{MHz}$ & $1 \mathrm{mJy}$ & $43^{\prime \prime} \times 43^{\prime \prime} \operatorname{cosec} \delta$ & 6 \\
NVSS & VLA & $1.4 \mathrm{GHz}$ & $0.45 \mathrm{mJy}$ & $45^{\prime \prime}$ & 4 \\
PMN & Parkes & $4850 \mathrm{MHz}$ & $5 \mathrm{mJy}$ & $4.2^{\prime}$ & 7 \\
\hline
\end{tabular}

Table 1: The equivalent surface brightness detection limit of surveys computed at a frequency of $1.4 \mathrm{GHz}$. ZA denotes zenith angle, and FWHM is the full width at half maximum of the telescope beam. 


\section{The ATLBS survey}

The new radio survey covers 8.4 square degrees of sky with a surface brightness sensitivity good enough to detect, at 5- $\sigma$, arcmin-scale extended sources with flux density down to $0.4 \mathrm{mJy}$. The survey has been given the acronym ATLBS - the Australia Telescope Low Brightness Survey. In Fig. 1 we compare the 5- $\sigma$ limiting surface brightness sensitivity equivalent at $1.4 \mathrm{GHz}$ for the different surveys versus angular size; ATLBS is about a factor 5 better in surface brightness sensitivity compared to any previous survey with comparable resolution.

High fidelity surveys for extended sources with low surface brightness requires good spatial frequency coverage. Holes in the $\mathrm{u}, \mathrm{v}$-coverage effectively reduce the number of independent synthesized beam areas within the primary beam and, consequently, confusion owing to discrete sources limits the image dynamic range and quality.

The ATLBS observations used the 750A, 750B, 750C and 750D array configurations of the E-W Australia Telescope Compact Array (ATCA). These together provide $4 \times 10=40$ baselines and because the ATCA antennas are $22-\mathrm{m}$ in diameter, the 40 spacings provide a nearly complete coverage over the $0-750 \mathrm{~m}$ range. At the 750-m baseline, Earth rotation moves the visibility point through $22 \mathrm{~m}$ in about $7 \mathrm{~min}$. Therefore, our observing strategy covered 19 fields in every 12-hr observing session: each field was observed for $20 \mathrm{sec}$ so that the 19 fields were re-visited once every 7 mins and each of the fields have, consequently, complete azimuthal coverage along the $\mathrm{u}, \mathrm{v}$-tracks. The blank time between successive pointings was about $3 \mathrm{sec}$ and this resulted in a 15\% loss of integration time. The 19 fields tile the sky in a hexagonal pattern, with pointings spaced 28.6 arcmin on the sky so as to cover the sky with uniform sensitivity.

In this special mode, every field was observed at a centre frequency of $1388 \mathrm{MHz}$ using a pair of $128-\mathrm{MHz}$ bands with dual polarization. The synthesized beam FWHM is 50 arcsec for naturally

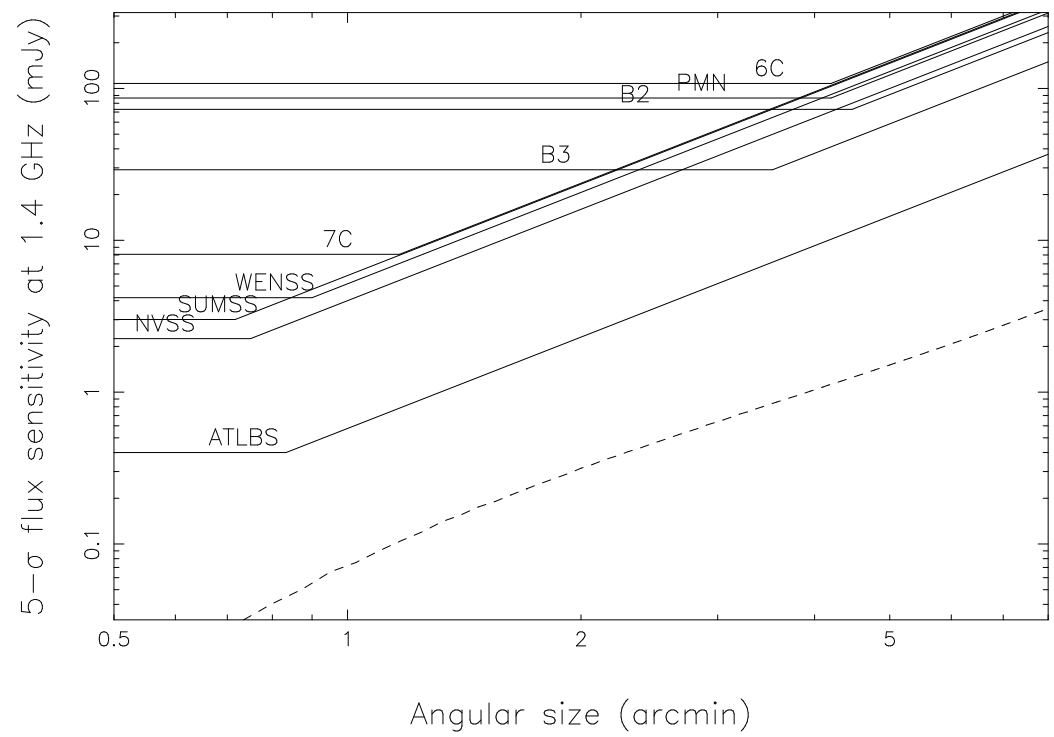

Figure 1: Limiting surface-brightness sensitivity (equivalent at $1.4 \mathrm{GHz}$ ) for various surveys versus source angular size. The survey resolution and flux sensitivity for earlier surveys are as listed in Table 1 . The dashed line has been drawn at 5 times the R.M.S. confusion noise for a fully-filled visibility sampling, and shows that the ATLBS survey is well above the confusion limit. 
weighted data. The observations are in full polarization mode resulting in full polarization images. The images have R.M.S. noise of $80 \mu \mathrm{Jy}_{\text {beam }}{ }^{-1}$, and the $5-\sigma$ detection limit is $0.4 \mathrm{mJy}_{\text {beam }}{ }^{-1}$.

It may be noted here that we have not truly mosaic imaged the fields: what we have done is a 'cut-and-paste' mosaic of a significant sky area with exceptional (and uniform) surface brightness sensitivity; however, the survey is relatively insensitive to sources with angular extents near the telescope primary beam FWHM size of $35^{\prime}$. The four 750-m arrays will yield images with about $1^{\prime}$ resolution; however, the visibility coverage is complete so good quality images with lower resolution may be synthesized from the data. The good u,v coverage enables imaging all structures up to $10^{\prime}$ in size with the $5-\sigma$ surface brightness sensitivity of 0.4 mJy beam ${ }^{-1}$.

The ATCA observations in the 750-m arrays include baselines to a ' $6-\mathrm{km}$ antenna': the long baselines to this antenna provides the high resolution that identifies compact components so that the presence of any cores and/or hotspots of extragalactic double sources would be distinguished from the extended source components. The long spacings are crucial in distinguishing the cases where closely spaced point sources appear as slightly resolved 'extended' sources in the images with $1^{\prime}$ beams.

We would like to emphasize the importance of the complete $\mathrm{u}, \mathrm{v}$-coverage that is possible with the ATCA in the mode described above. Good and complete visibility coverage is vital to avoid confusion from the stronger sources above the detection limit. If the $\mathrm{u}, \mathrm{v}$-coverage were a factor of two less well filled, the effective synthesized beam area that would respond to weak source confusion would double, and the R.M.S. confusion owing to the weak sources would double. The complete $\mathrm{u}, \mathrm{v}$-coverage that may be achieved with the fast position switching possible at the ATCA is crucial to confusion-free imaging at these low-surface-brightness levels.

\section{Preliminary results}

Two regions-each a mosaic of 19 fields-have been observed centred at RA: $00^{\mathrm{h}} 35^{\mathrm{m}} 00^{\mathrm{s}}$, DEC: $-67^{\circ} 00^{\prime} 00^{\prime \prime}$ and RA: $00^{\mathrm{h}} 59^{\mathrm{m}} 17^{\mathrm{s}}$, DEC: $-67^{\circ} 00^{\prime} 00^{\prime \prime}$ (J2000 epoch), covering a total of $8.4 \mathrm{deg}^{2}$ sky area. The final synthesized mosaic images, made with beam FWHM of $50^{\prime \prime}$, have no artefacts above 3- $\sigma$ of the thermal noise: the dynamic range-defined as the ratio of the peak intensity to the peak artefact anywhere in the image-exceeds 1000. A portion of the survey is shown in Fig. 2. Thus, we reliably detect and interpret contours at 5- $\sigma\left(0.4 \mathrm{mJy}^{\text {beam }}{ }^{-1}\right)$. Additionally, by constraining the deconvolution algorithm to detect sources in the regions where the low surface brightness survey identified sources, we have successfully used the baselines to the '6-km antenna' to independently image the compact components in all of the sources with a 5" FWHM beam.

An immediate and obvious result of the survey, which demonstrated its quality, was the discovery of a 1.9-Mpc giant radio galaxy J0034-6639 (seen in Fig. 2). What is remarkable is that the lobe surface brightness is lower than any other giant radio galaxy known to date and has a value similar to that of the diffuse radio halo in the Coma cluster; nevertheless, J0034-6639 is detected in ATLBS at $40-\sigma$.

We detect 1094 sources exceeding 0.4 mJy. At $50^{\prime \prime}$ resolution, $10 \%$ of the sources have size exceeding 1.5 times the beam area, indicating that a significant population of extended sources exist at mJy flux-density levels. 500 sources exceed $1 \mathrm{mJy}$ : this sample represents a complete 


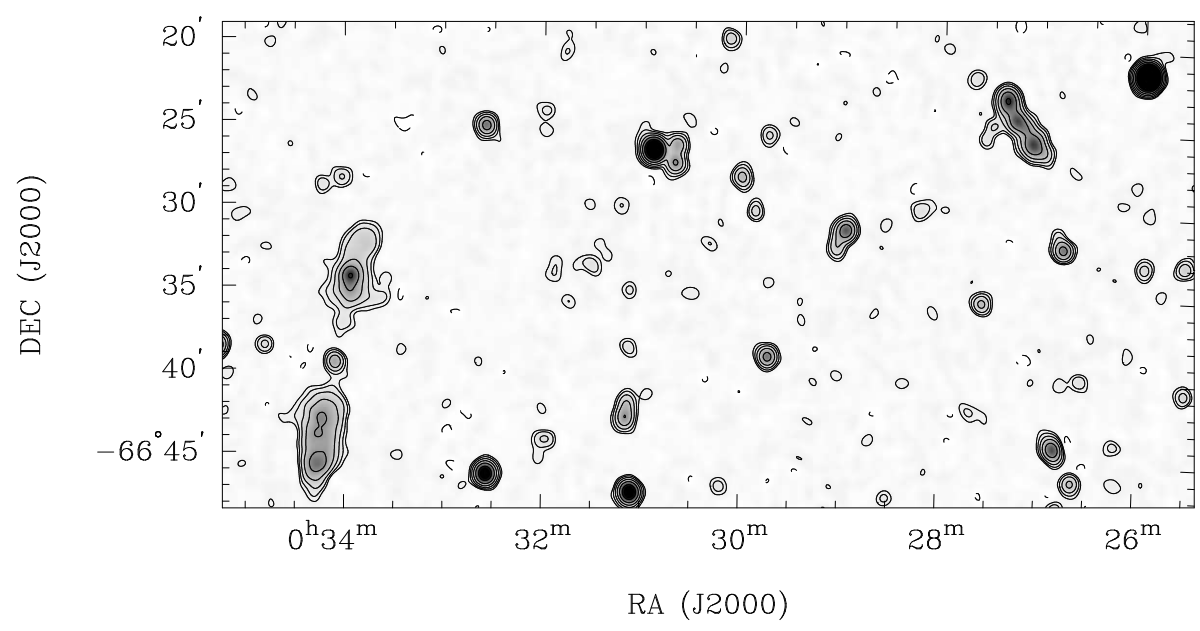

Figure 2: Portion of the ATLBS survey. The image was made with beam FWHM $52.4 \times 47.4 \operatorname{arcsec}^{2}$ at position angle $6^{\circ}$. Contours at $0.25 \mathrm{mJy}^{\text {beam }}{ }^{-1} \times(-2,-1,1,2,4,8,16,32,64,128)$.

flux-limited sample because it includes extended sources whose peak may be as low as $0.4 \mathrm{mJy}$. 145 of these sources, or $30 \%$ of the 1 -mJy sample, have more than $50 \%$ of the total flux density in extended emission that is detected in the low-surface-brightness images with $50^{\prime \prime}$ beam, but missing in the $5^{\prime \prime}$ resolution images.

A few extended radio sources are coincident with nearby interacting and star-forming systems, a few are halo-type systems identified with faint clusters of galaxies. Largely they are extragalactic radio sources associated with AGN-type activity: radio cores are often detected that contain a large fraction of the total flux density; however, a significant number have a large fraction in extended emission. Some extended sources have radio cores detected coincident with optical counterparts in digital sky survey (DSS) images, some have radio cores but no obvious optical IDs in DSS, some have optical IDs close to their centroids but no core detected in the higher resolution images.

\section{Ongoing work}

A first paper presenting that ATLBS low-surface-brightness images and initial results is under preparation. Higher resolution radio observations with the ATCA is underway to image the structures of all the extended mJy sources. We are also mosaic imaging the fields in the $\mathrm{K}_{\mathrm{s}} \mathrm{NIR}$ band with the 3.9-m Anglo-Australian Telescope, to improve identifications.

\section{Acknowledgments}

The ATCA is part of the Australia Telescope, funded by the Commonwealth of Australia for operation as a National Facility managed by CSIRO.

\section{References}

[1] R. Saunders, J.E. Baldwin, P.J. Warner, A totally incomplete sample of extended 6C radio sources, 1987 MNRAS 225713. 